\title{
Data-mining of potential antitubercular activities from molecular ingredients of Traditional Chinese Medicines
}

Background Traditional Chinese medicine encompasses a well established alternate system of medicine based on a broad range of herbal formulations and is practiced extensively in the region for the treatment of a wide variety of diseases. In recent years, several reports describe in depth studies of the molecular ingredients of Traditional Chinese Medicines on the biological activities including anti-bacterial activities. The availability of a well-curated dataset of molecular ingredients of Traditional Chinese Medicines and accurate in-silico cheminformatics models for data mining for antitubercular agents and computational filters to prioritize molecules has prompted us to search for potential hits from these datasets.

Results We used a consensus approach to predict molecules with potential antitubercular activities from a large dataset of molecular ingredients of Traditional Chinese Medicines available in the public domain. We further prioritized 160 molecules based on five computational filters (SMARTSfilter) so as to avoid potentially undesirable molecules. We further examined the molecules for permeability across Mycobacterial cell wall and for potential activities against non-replicating and drug tolerant Mycobacteria. Additional in-depth literature surveys for the reported antitubercular activities of the molecular ingredients and their sources were considered for drawing support to prioritization.

Conclusions Our analysis suggests that datasets of molecular ingredients of Traditional Chinese Medicines offer a new opportunity to mine for potential biological activities. In this report, we suggest a proof-of-concept methodology to prioritize molecules for further experimental assays using a variety of computational tools. We also additionally suggest that a subset of prioritized molecules could be used for evaluation for tuberculosis due to their additional effect against non-replicating tuberculosis as well as the additional hepatoprotection offered by the source of these ingredients. 


\title{
1 Data-mining of potential antitubercular activities from 2 molecular ingredients of Traditional Chinese 3 Medicines
}

4 Salma Jamal, Open Source Drug Discovery Consortium, Vinod Scaria ${ }^{\$}$

\author{
$5 \quad{ }^{1}$ CSIR Open Source Drug Discovery Unit, Anusandhan Bhavan, 2 Rafi Marg, Delhi \\ $6 \quad 110001$ \\ $7 \quad{ }^{2}$ GN Ramachandran Knowledge Center for Genome Informatics, CSIR Institute of \\ 8 Genomics and Integrative Biology (CSIR-IGIB), Mall Road, Delhi 110007, India \\ 9 \$Address for correspondence: vinods@igib.in
}




\section{Background}

12 Traditional Chinese medicine encompasses a well established alternate system of 13 medicine based on a broad range of herbal formulations and is practiced extensively in 14 the region for the treatment of a wide variety of diseases. In recent years, several reports describe in depth studies of the molecular ingredients of Traditional Chinese Medicines on the biological activities including anti-bacterial activities. The availability of a well-curated dataset of molecular ingredients of Traditional Chinese Medicines and accurate in-silico cheminformatics models for data mining for antitubercular agents and computational filters to prioritize molecules has prompted us to search for potential hits from these datasets.

21 Results

We used a consensus approach to predict molecules with potential antitubercular activities from a large dataset of molecular ingredients of Traditional Chinese Medicines available in the public domain. We further prioritized 160 molecules based on five computational filters (SMARTSfilter) so as to avoid potentially undesirable molecules. We further examined the molecules for permeability across Mycobacterial cell wall and for potential activities against non-replicating and drug tolerant Mycobacteria. Additional in-depth literature surveys for the reported antitubercular activities of the molecular ingredients and their sources were considered for drawing support to prioritization.

Conclusions

Our analysis suggests that datasets of molecular ingredients of Traditional Chinese Medicines offer a new opportunity to mine for potential biological activities. In this report, we suggest a proof-of-concept methodology to prioritize molecules for further experimental assays using a variety of computational tools. We also additionally suggest that a subset of prioritized molecules could be used for evaluation for tuberculosis due to their additional effect against non-replicating tuberculosis as well as the additional hepato-protection offered by the source of these ingredients.

Keywords:

39 Tuberculosis, Traditional Chinese Medicine, Cheminformatics, Virtual Screening, Data-

40 mining 


\section{Introduction}

42 Traditional Medicine still forms the mainstay of healthcare in many parts of the world.

43 Traditional Chinese Medicine (TCM) is one of the well developed and established 44 systems of traditional medicine, and largely followed in some parts of Eastern Asia 45 where it forms one of the major alternative medicinal practices [1]. TCM as a system of 46 medicine was, founded almost 2000 years ago and is dependent on the concepts of five 47 elements and guided by the Chinese philosophy of Ying and Yang [2, 3]. Recently, 48 efforts have been underway to investigate the practice of TCM using molecular 49 approaches. This has led to the identification and molecular characterization of 50 ingredients used in Traditional Chinese Medicines [4, 5]. These efforts have led to the 51 systematic curation of the molecular structures and the biological activities of ingredients 52 of Traditional Chinese Medicines [6-9]. In addition, molecular basis of the action and 53 mechanisms of modulation [10,11], immunomodulatory and antimicrobial activities of

54 Traditional Chinese Medicines have also been actively pursued [12, 13].

55 Tuberculosis is considered one of the major tropical diseases, caused by intracellular 56 pathogen Mycobacterium tuberculosis. According to the World Health Organization 57 (WHO) Global Tuberculosis Report 2012, Tuberculosis causes over 1.4 million deaths 58 annually worldwide and a major cause of morbidity and mortality especially in the developing countries in Asia and Africa [14]. The paucity of new drugs for the treatment of Tuberculosis along with the rampant and unprecedented rise of drug-resistant strains made it imperative to discover potential new drugs for tuberculosis [15]. The conventional process of drug discovery involves screening of large molecular libraries of molecules for biological activities, and it is a tedious, expensive and time-consuming process [16]. Data mining approaches based on cheminformatics modeling has been extensively used to prioritize molecules from large chemical datasets for specific biological activities. Such in-silico prioritization of molecules has been suggested to accelerate drug discovery by drastically reducing the time and cost-factor in conventional drug discovery processes [17-20].

Cheminformatics and data mining approaches have been used to mine biological activities from molecular data sets of ingredients in traditional Chinese Medicines [21, 22]. The availability of large molecular databases with systematically curated molecular data, sources and activities of ingredients of Traditional Chinese Medicines offer a new opportunity to use advanced data-mining tools to mine for potential activities, especially for pathogens causing neglected tropical diseases [6-9]. Previously we used highthroughput bioassay data sets to create highly accurate data-mining classifiers based on machine learning of molecular properties including antimicrobial activities for a number

77 of neglected tropical diseases including Tuberculosis, and Malaria [23-25]. 
In the present report, we used one of the largest and well characterized compilation of molecular ingredients in traditional Chinese Medicine and applied a host of previously generated cheminformatics models aimed at identifying potential hits with antitubercular activity against Tuberculosis. We additionally employed methodologies for filtering out potential molecules using a series of in-silico filters. Our analysis revealed a total of 19 hits for antitubercular activity from the dataset. In-depth literature survey suggests 4 of these molecules are derived from plant products known to be used against tuberculosis, suggesting that the computational approach can be immensely useful in identifying and characterizing molecular activities. To the best of our knowledge, this is the first and most comprehensive data-mining and cheminformatic analysis of potential antitubercular agents from traditional Chinese medicine ingredients.

\section{Materials and Methods}

\section{Data Sets}

Molecular Data Sets of ingredients of Traditional Chinese Medicines were retrieved from Traditional Chinese Medicines Integrated Database (TCMID) [27]. TCMID constitutes one of the most comprehensive online resources for ingredients used in TCM. The database hosts information on over 25, 210 pure molecules retrieved from literature and other data resources.

\section{Computational models for antitubercular activity}

The computational predictive models used in our analysis were based on the following two confirmatory screens conducted to identify novel inhibitors of Mycobacterium tuberculosis H37Rv, previously published by our group [23, 24]. The computational models used are available online at http://vinodscaria.rnabiology.org/2C4C/models.

Briefly these models were based on two bioassays deposited in PubChem and carrying IDs AID 1332 and AID 449762. Both the assays were based on microdilution Alamar Blue assays. The former used $7 \mathrm{H} 12$ broth while the latter used $7 \mathrm{H} 9$ media. A total of 1,120 and 327, 669 compounds were screened in the respective assays. The models were generated using a machine learning approach as described in Periwal et al and Periwal et al [23, 24]. For the AID 1332 assay model was generated based on the Random forest classification algorithm and was evaluated using a variety of statistical measures which include accuracy, Balanced Classification Rate (BCR) and Area under Curve (AUC). Balanced Classification Rate is an average of sensitivity and specificity which introduces a balance in the classification rate. The model had an accuracy of $82.57 \%, B C R$ value of $82.2 \%$ and AUC value of 0.87 . The AID 449762 assay model was generated based on SMO (Sequential Minimization Optimization) algorithm and was found to be $80.52 \%$ accurate, with BCR value of $66.30 \%$ and AUC as 0.75 . 
114 In addition, we created an additional model to predict the molecules active against non-

115 replicating drug tolerant Mycobacterium tuberculosis. The assay was deposited in

116 PubChem with identifier AID 488890. A total of 3, 24, 437 compounds were screened for

117 the activity. The model was generated using Random forest classification algorithm as

118 described in the previous papers [23-26] and had an accuracy of $76 \%$, BCR value

$11985.2 \%$ and AUC 0.66.

\section{Molecular Descriptors}

121 Molecular descriptors for each of the molecules were computed using PowerMV [28], 122 popular cheminformatics software widely used to compute molecular descriptors. A total 123 of 179 molecular descriptors were computed for each molecule. Out of the total 179 124 molecular descriptors, a few descriptors were pruned using bespoke scripts written in 125 Perl depending on whether they were used in creating the respective models. We 126 pruned a total of 29 and 25 descriptors corresponding to AID 1332 and AID 449762 respectively, while 25 were pruned for the AID 488890 model.

\section{Formats and Format conversion}

The molecules were downloaded in mol2 format and converted to SDF (Structural Data Format) format using Openbabel [29]. The molecular descriptors were converted to ARFF format compatible with Machine learning toolkit Weka [30]. We used custom scripts written in Perl for the format conversions. A complete list of scripts is also available at Crowd Computing for Cheminformatics $(2 \mathrm{C} 4 \mathrm{C})$ repository at URL: http://vinodscaria.rnabiology.org/2C4C/models.

135

136

137

138

139

140

141

\section{2}

143

144

145

146

147

148

149

150

\section{SMARTS filters}

The SMARTS filter is employed to remove the molecules with fragments leading to toxicity or unwanted reactivity. We used a set of SMARTS filters for the consensus candidate anti-tubercular molecules. The online server SMARTSfilter (http://pasilla.health.unm.edu/tomcat/biocomp/smartsfilter) web application was used for all comparisons. The web application was used to filter out molecules, which match to any of the five undesirable SMARTS catalogs.

\section{Mycobacterium tuberculosis permeability prediction}

The small molecules could not be effective unless they are able to penetrate the cell wall. Recent computational tool, MycPermCheck [31], to predict permeability of small molecules across Mycobacterium tuberculosis was employed to filter the subset of potential active molecules.

\section{Data Mining}

We used Weka, a popular and freely available Data Mining Software toolkit. Predictions were performed for the dataset across the two models corresponding to assays AID 1332 and AID 449762 independently. Further, molecules predicted active in both the 
151 datasets were collated and analyzed for additional properties including activity against 152 non-replicating drug tolerant Mycobacterium tuberculosis and potential to permeate the 153 Mycobacterium tuberculosis cell wall. Additional filters which discount molecules with 154 toxic fingerprints were removed using SMARTS filters. The summary of the entire 155 workflow of prioritization is depicted as a Schema (Figure 1).

\section{Results}

\section{Summary of Datasets and Molecules}

158 A total of 25,210 ingredients were downloaded from Traditional Chinese Medicines 159 Integrated Database (TCMID). We could retrieve molecular information for only 12,018 160 of the ingredients in the form of SMILE notations and the rest were not considered for 161 further analysis. The molecules considered along with their SMILES are detailed in 162 Supplementary Table 1. A total of 179 descriptors were calculated using PowerMV as described above. The descriptors were further pruned for each of the models as described in the Materials and Methods section using custom scripts in Perl. This corresponds to 150 and 154 descriptors respectively for models AID 1332 and AID 449762 and 154 for AID 488890. The models, descriptors and scripts for formatting the files are available at the Crowd Computing for Cheminformatics Model Repository [http://vinodscaria.rnabiology.org/2C4C/models].

\section{Prediction of potential anti-tubercular hits} The 12, 018 molecules obtained from TCMID were analyzed for the antitubercular activity using the computational predictive models as described above. The AID 1332 and AID 449762 models predicted 2, 363 compounds and 5, 864 compounds respectively as potentially active anti-tubercular. Of these molecules, a total of 1,472 molecules were predicted potential actives by both the models based on molecular descriptors and were considered for further analysis (Supplementary Table 2). Briefly we used a popular approach for filtering molecules with undesirable properties. These included briefly using SMARTS filters. Molecules which passed the filtering step were further evaluated for their effect against drug-tolerant and slow growing Mycobacterium. Molecules were further evaluated for their potential permeability with respect to the Mycobacterial cell wall.

\section{SMARTS filter for filtering undesirable structures}

182 We used a set of five SMARTS filters to remove the molecules matching to any of these 183 filters. Such substructure based filtering approach has been extensively used to 184 prioritize molecules by filtering unwanted or potential false positives in cheminformatics 185 screens [32]. The SMARTS filters included 5 independent approaches namely Glaxo, 186 PAINS, Oprea, Blake and ALARM-NMR used in tandem. Pan Assay Interference 187 Compounds (PAINS) describes a set of substructures known to be promiscuous and 188 have issues in high throughput assays [33], while the Glaxo filter describes unsuitable 
189

190

191

192

193

194

195

196

197

198

199

200

201

202

203

204

205

206

207

208

209

210

211

212

213

214

215

216

217

218

219

220

221

222

223

224

225

hits or unsuitable natural products [34]. ALARM NMR assay to detect reactive molecules by nuclear magnetic resonance (ALARM-NMR) set filters for molecules which are reactive false positives in high-throughput assays by oxidizing or alkylating a protein target [35]. The Glaxo, Oprea and Blake filters were based on specific fitness properties. The Glaxo method involves classification of the molecules into different chemical categories based on the presence of acids, bases, electrophiles and nucleophiles in the molecule. Prior to the categorization the molecules are filtered for non-drug like properties and to remove inappropriate functional groups (unsuitable leads and unsuitable natural products) [34].

Out of a total of 1472 molecules, 160 molecules passed all the filters. A total of $63.1 \%$ (929) molecules failed the ALARM NMR filter, while 49.9\% (734) failed to pass Oprea filter. Similarly $49 \%$ (722) failed to pass the PAINS filter. The detailed schema showing the number of molecules failed by each filter is depicted in Figure 3. A similar comparison of the complete set of 12, 018 TCMID compounds revealed that only 1,539 compounds passed all the filters. We observed that most of the molecules did not pass through ALARM NMR $(60.7 \%, 7,295)$ molecules followed by Oprea filter $(52.4 \%, 6,303)$ molecules and $5,799,48.3 \%$ molecules could not pass through PAINS filter.

Molecules potentially active against non-replicating drug tolerant Mycobacterium tuberculosis.

A total of 160 compounds filtered through SMARTSfilter were tested using a computational predictive model for potential activity against non-replicative Mycobacterium tuberculosis. The model predicted 19 compounds as active to act as potential inhibitors of non-replicating drug tolerant Mycobacterium tuberculosis. The detailed description about 19 compounds is given in Table 1. The table also shows the permeability probability of the molecules to pass through Mtb cell wall.

Pharmacophore Search in 19 molecules found to be potentially active against non-replicating drug tolerant Mycobacterium tuberculosis.

Since pharmacophore represents the features which play a key role in the recognition of ligand by the target molecule, we generated the pharmacophore features for the 19 molecules identified to be active against non-replicating drug tolerant Mycobacterium. tuberculosis using PharmaGist software [36]. PharmaGist computes the pharmacophore model by doing multiple flexible alignments of the input molecules. We have reported the three highest-scoring pharmacophores which can be used for the discovery of novel drug entities (Figure 3).

Mycobacterium tuberculosis permeability prediction

We employed the MycPermCheck a recently published methodology to predict molecular permeability to Mycobacterial cell wall to estimate the potential permeability of 
the prioritized molecules. All the 160 molecules which passed the five SMARTSfilters were further evaluated for their ability to penetrate Mtb cell wall. Analysis revealed 9 molecules with highest probability $(>0.98)$ to permeate Mycobacterium cell wall barrier (Supplementary Table 3).

\section{Literature search suggests evidence of the sources and molecules used with antitubercular properties}

We further searched for the role of the plant sources of the molecules in regard to their use or known information on antibacterial or anti tubercular activities. We found several molecules herbs to have antitubercular effects. These are Petasites japonicus [37], Piper trichostachyon [38], Solanum torvum [39], Fritillaria przewalskii [40], Hernandia sonora [41] and Phyllanthus urinari [12]. In addition, many of the herbs have been shown to have hepatoprotective activities, which include Annona reticulata [42, 43], Annona squamosa [42, 43], and Camellia sinensis [44]. This offers a new opportunity for new drug development considering that most of the established first-line drugs used in the treatment of tuberculosis are hepatotoxic $[45,46]$. We also found the molecules, Hinokiol [47], Totarol [48], Murrayafoline a [49] and 2-hexenyl benzoate [50] have been known to show antitubercular effects. The 9 molecules which were identified by MycPermCheck, to be able to penetrate the cell wall of Mycobacterium tuberculosis are listed in Supplementary Table 3. We tried to find out if any of these molecules have been observed to show some antitubercular or antimicrobial activity and we found out 6 molecules that include Thalfinine [9], Azulene [51], Erypoegin e [52], Pongapinone a [53], Achilleol c [54] and Murrayafoline a [49].

\section{Discussion and Conclusions}

Traditional Chinese Medicine (TCM) has been a major alternative medicine practice, widely followed in many parts of China and Southeast Asia [1]. Enormous efforts in the recent years have been invested in the systematic identification and characterization of the molecular activities of the ingredients and scientific validation of their effects [10, 11]. The availability of well curated databases of ingredients of Traditional Chinese Medicines has opened up new avenues for molecular screening as well as in-silico studies, including target-based docking [6-9]. In depth screens of Chinese Medicine derived compounds have been performed for a variety of pathophysiologies, including cancer [55], inflammatory diseases [56, 57], cardiovascular diseases [58] and infections [59] etc, just to name a few. These databases are being extensively used for therapeutic development [60].

Our group has earlier used a machine learning based approach on publicly available high-throughput screen datasets to create highly accurate models for predicting specific molecular activities against pathogens causing Tuberculosis [23, 24] and Malaria [25]. 
Such accurate in-silico models offer a new opportunity to prioritize large molecular databases in silico, significantly reducing the failures, cost and effort. The availability of a well-curated database of molecular ingredients of traditional Chinese Medicines offer a new opportunity to mine potential active anti-tubercular agents and prioritize them for screening and in-depth functional assays.

268

269

270

271

272

273

274

275

276

277

278

279

280

281

282

283

284

285

286

287

288

289

290

291

292

293

294

295

296

297

298

299

300

In the present study, we have used two computational models based on high throughput assays on Mycobacterium tuberculosis. In addition to the predictive models, we used a filter based approach to filter out potential false positives/toxic molecules. Our analysis revealed a total of 1,472 molecules predicted active by both the models, of which 160 molecules passed all the five filters. These molecules were further evaluated for their permeability to mycobacterial cell wall and potential additional activity on drug-tolerant and non-replicating Mycobacterium tuberculosis. We also further show evidence from literature that these molecules or their sources have been used in the treatment of therapeutics. This study is not without caveats; the primary one being that the consensus approach used in the present study could be over-stringent so as to miss out on potential antitubercular hits from the screening approach. The second, being that the findings would require re-screening and in-depth functional analysis. Nevertheless we show from independent evidence that molecular ingredients or sources of the prioritized molecules have been extensively used as antibacterial or specifically in the treatment of tuberculosis. In the present study we show a proof-of-concept that data-mining approaches using accurate cheminformatics models could possibly be used to mine large datasets and prioritize molecules for antitubercular screening.

Our analysis suggests that molecular ingredients of Traditional Chinese Medicines offer an attractive starting point to mine for potential antitubercular agents. Chinese Medicines alone [61] or in combination [62] with western medicine have been explored for the treatment of tuberculosis. Potential use of Chinese Medicines in combination with the standard antitubercular drugs could be an attractive alternative that could be explored in much detail. There is ample evidence in published literature that some of the ingredients of the short-listed antitubercular molecules have additional hepatoprotective action, which could be effectively used in the background of hepatotoxicity induced by the first line of drugs. We also suggest that 19 of the prioritized molecules have additional activity against drug-tolerant and non-replicating Mycobacterium tuberculosis suggesting that they could be potentially developed into leads for Multidrug resistant and latent tuberculosis. We hope that this report would accelerate in in-depth analysis and discovery of anti-tubercular agents from molecular ingredients of Traditional Chinese Medicines.

\section{Competing interests}

\footnotetext{
The authors declare that they have no competing interests.
} 


\section{Authors' contributions}

302 SJ under the supervision of VS carried out the analysis and reviewed the results.

303 OSDDC supported the work through regular discussions and funding. Both authors

304 wrote, reviewed and approved the final manuscript.

305

306

307

308

309

310

311

\section{Acknowledgements}

The authors thank Ms Vinita Periwal for sharing the models for antitubercular activities and for discussions. Authors also thank Dr S Ramachandran and Dr Sridhar Sivasubbu for reviewing the manuscript and suggestions. The help and support from the National Knowledge Network (NKN) and the CDAC-Garuda grid for the connectivity and access to the compute facility is acknowledged. The Open Source Drug Discovery Consortium members are the registered members on http://sysborg2.osdd.net

\section{References}

1. Ooi GL: Chinese medicine in Malaysia and Singapore: the business of healing. Am J Chin Med. 1993, 21(3-4):197-212.

2. Qiu J: Traditional medicine-a culture in the balance. Nature 2007, 448:126-128.

3. Normile D: Asian medicine. The new face of traditional Chinese medicine. Science 2003, 299: 188-190.

4. Wang MW, Hao X, Chen K: Biological screening of natural products and drug innovation in China. Philos. Trans. Roy. Soc. Lond. B Biol. Sci. 2007, 362:10931105.

5. Sucher NJ: Insights from molecular investigations of traditional Chinese herbal stroke medicines: implications for neuroprotective epilepsy therapy. Epilepsy Behav. 2006, 8(2):350-62.

6. Chen X, Zhou H, Liu YB, Wang JF, Li H, Ung CY, Han LY, Cao ZW, Chen YZ: Database of traditional Chinese medicine and its application to studies of mechanism and to prescription validation. Br. J. Pharmacol. 2006, 149:10921103.

7. Fang YC, Huang HC, Chen HH, Juan HF: TCMGeneDIT: a database for associated traditional Chinese medicine, gene and disease information using text mining. BMC Complement. Altern. Med. 2008, 8:58. 
331 8. Chen CYC: TCM Database@Taiwan: the world's largest traditional Chinese 332 medicine database for drug screening in silico. Plos One 2011, 6:e15939.

9. Zhou J, Xie G, Yan X: (eds) Encyclopedia of Traditional Chinese Medicines Molecular Structures, Pharmacological Activities, Natural Sources and Applications. Springer: New York; 2011.

10. Li XM, Brown L: Efficacy and mechanisms of action of traditional Chinese medicines for treating asthma and allergy. Journal of Allergy and Clinical Immunology 2009, 123(2):297-306.

11. Wen Z, Wang Z, Wang S, Ravula R, Yang L, et al:: Discovery of Molecular Mechanisms of Traditional Chinese Medicinal Formula Si-Wu-Tang Using Gene Expression Microarray and Connectivity Map. PLOS ONE 2011, 6(3):e18278.

12. Nair RR, Abraham RS: Integrating the Science of Pharmacology and Bio Informatics Phyllanthus "The wonder plant". Advanced Biotech January 2008.

13. Nader LA, de Mattos AA, Picon PD, Bassanesi SL, De Mattos AZ, Pineiro Rodriguez $M$ : Hepatotoxicity due to rifampicin, isoniazid and pyrazinamide in pateints with tuberculosis: is anti-HCV a risk factor? Ann Hepatol. 2010, 9(1):70-74.

15. Shah NS, Wright A, Bai GH, Barrera L, Boulahbal F, et al.: Worldwide emergence of extensively drug-resistant tuberculosis. Emerg Infect Dis. 2007, 13(3):380-7.

16. DiMasi, JA, Hansen, RW, Grabowski, HG: The price of innovation: new estimates of drug development costs. Journal of Health Economics 2003, 22:151-185.

17. Vert JP, Jacob L: Machine learning for in silico virtual screening and chemical genomics: new strategies. Comb Chem High Throughput Screen 2008, 11:677685.

18. Melville JL, Burke EK, Hirst JD: Machine Learning in Virtual Screening. Comb Chem High Throughput Screen 2009, 12:332-343.

19. Schierz AC: Virtual screening of bioassay data. J Cheminform 2009, 1:21. machine learning techniques. Drug Metab Dispos 2009, 37:658-664. 
362 21. Li XJ, Kong DX, Zhang HY: Chemoinformatics approaches for traditional 363 Chinese medicine research and case application in anticancer drug discovery. 364 Curr Drug Discov Technol. 2010, 7(1):22-31.

365 22. Zhang K, Li Y, Zhang ZR, Guan WH, Pu YC: Chemoinformatics study on 366 antibacterial activity of traditional Chinese medicine compounds. Zhongguo 367 Zhong Yao Za Zhi. 2013, 38(5):777-80.

368 23. Periwal V, Rajappan JK, Jaleel AU, Scaria V: Predictive models for anti-tubercular 369 molecules using machine learning on high-throughput biological screening datasets. BMC Res Notes 2011, 4:504.

24. Periwal V, Kishtapuram S, Scaria V: Computational models for in-vitro antitubercular activity of molecules based on high-throughput chemical biology screening datasets. BMC Pharmacol 2012, 12:1.

25. Jamal S, Periwal V, Scaria V: Predictive modeling of anti-malarial molecules inhibiting apicoplast formation. BMC Bioinformatics 2013, 14:55.

26. Jamal S, Periwal V, Scaria V: Computational analysis and predictive modeling of small molecule modulators of microRNA. Journal of Cheminformatics 2012, 4:16.

27. Ruichao Xue, Zhao Fang, Meixia Zhang, Zhenghui Yi, Chengping Wen, Tieliu Shi: TCMID: traditional Chinese medicine integrative database for herb molecular mechanism analysis. Nucleic Acids Research 2013, 41:D1089-D1095.

28. Liu K, Feng J, Young SS: PowerMV: a software environment for molecular viewing, descriptor generation, data analysis and hit evaluation. $J$ Chem Inf Model 2005, 45:515-522.

29. O'Boyle NM, Banck M, James CA, Morley C, Vandermeersch T, Hutchison GR: Open

30. Bouckaert RR, Frank E, Hall MA, Holmes G, Pfahringer B, Reutemann P: Weka -Experiences with a Java Open-Source Project. Journal of Machine Learning Research 2010, 2533-2541.

31. Merget B, Zilian D, Müller T, Sotriffer CA: MycPermCheck: The Mycobacterium tuberculosis permeability prediction tool for small molecules. Bioinformatics 2013, 29(1): 62-68.

32. Singla D, Tewari R, Kumar A, Raghava GP: Open Source Drug Discovery Consortium. Designing of inhibitors against drug tolerant Mycobacterium tuberculosis (H37Rv). Chem Cent J. 2013, 7(1): 49. 
33. Baell JB, Holloway GA: New Substructure Filters for Removal of Pan Assay Interference Compounds (PAINS) from Screening Libraries and for their Exclusion in Bioassays. J. Med. Chem 2010, 53:2719-2740.

34. Hann M, Hudson B, Lewell X, Lifely R, Miller L, Ramsden N: Strategic pooling of compounds for high-throughput screening. J Chem Inf Comput Sci. 2009, 39(5):897-902.

401

35. Huth JR, Mendoza R, Olejniczak ET, Johnson RW, et al:: ALARM NMR: A Rapid and Robust Experimental Method to Detect Reactive False Positives in Biochemical Screens. J. Am. Chem. Soc. 2005, 127:217-224.

36. Schneidman-Duhovny D, Dror O, Inbar Y, Nussinov R, Wolfson HJ: PharmaGist: a webserver for ligand-based pharmacophore detection. Nucleic Acids Research 2008, 36:W223-8.

37. John EF: A Barefoot Doctors Manual: The American Translation of the Official Chinese Paramedical Manual. 1999.

38. Wagner H, Wolff P: New Natural Products and Plant Drugs with Pharmacological, Biological or Therapeutical Activity. Springer-Varlag Berlin; 1977:p212.

39. Silva KN, Silva RC, Coelho VPM, Agra M: A pharmacobotanical study of vegetative organs of Solanum torvum. Brazilian Journal of Pharmacognosy 2011, 21(4):568-574.

40. Chang HM, Paul PH: Pharmacology and Applications of Chinese Materia Medica. 2001.

41. Udino L, Abaul J, Bourgeois P, Gorrichon L, Duran H, Zedde C: Lignans from the Seeds of Hernandia sonora. Planta Med. 1999, 65(3):279-81.

42. Thattakudian Sheik Uduman MS, Sundarapandian R, Muthumanikkam A, et al.: Protective effect of methanolic extract of Annona squamosa Linn in isoniazid rifampicin induced hepatotoxicity in rats. Pak J Pharm Sci. 2011, 24(2):129-34.

43. Mohamed Saleem TS, Christina AJM, Chidambaranathan N, Ravi V: Hepatoprotective activity of Annona squamosa Linn. on experimental animal model. Int J Pharm Pharm Sci. 2008, 1(3):1-7.

44. Issabeagloo E, Taghizadieh M: Hepatomodulatory Action of Camellia sinensis Aqueous Extract against Isoniazid-Rifampicin Combination Induced Oxidative Stress in Rat. Advances in Bioresearch 2012, 3:18-27. 
428

429

430

431

432

433

434

435

436

437

438

439

440

441

442

443

444

445

446

447

448

45. Yew WW, Leung CC: Antituberculosis drugs and hepatotoxicity. Respirology 2006, 11(6):699-707.

46. Liu Q, Garner P, Wang Y, Huang B, Smith H: Drugs and herbs given to prevent hepatotoxicity of tuberculosis therapy: systematic review of ingredients and evaluation studies. BMC Public Health 2008, 21(8):365.

47. Chen JJ, Wu HM, Peng CF, Chen IS, Chu SD: seco-Abietane diterpenoids, a phenylethanoid derivative, and antitubercular constituents from Callicarpa pilosissima. J Nat Prod. 2009, 72(2):223-8.

48. Jaiswal R, Beuria TK, Mohan R, Mahajan SK, Panda D: Totarol inhibits bacterial cytokinesis by perturbing the assembly dynamics of FtsZ. Biochemistry 2007, 46(14):4211-20.

49. Choi TA, Czerwonka R, Fröhner W, Krahl MP, Reddy KR, Franzblau SG, Knölker HJ: Synthesis and activity of carbazole derivatives against Mycobacterium tuberculosis. ChemMedChem 2006, 1(8):812-5.

50. He, Yantao, Li-fan ZENG, Zhong-Yin Zhang: Tyrosine phosphatase inhibitors and uses thereof to modulate the activity of enzymes involved in the pathology of mycobacterium tuberculosis. WIPO Patent Application PCT/US2012/035039.

51. Kurti V, Uldrich J: Effect of azulenes on Mycobacterium tuberculosis \& their use in the local treatment of tuberculous cystitis. Cas Lek Cesk. 1958, 97(3):67-71.

52. Sato M, Tanaka H, Tani N, Nagayama M, Yamaguchi R: Different antibacterial actions of isoflavones isolated from Erythrina poeppigiana against methicillinresistant Staphylococcus aureus. Lett Appl Microbiol. 2006, 43(3):243-8.

53. Rani SM, Dayanand CD, Shetty J, Vegi PK, Kutty AVM: Evaluation of Antibacterial Activity of Pongamia pinnata linn on Pathogens of Clinical Isolates. American Journal of Phytomedicine and Clinical Therapeutics 2013, 1(8):645-651.

54. linuma M1, Tosa H, Tanaka T, Kanamaru S, Asai F, Kobayashi Y, Miyauchi K, Shimano R: Antibacterial activity of some Garcinia benzophenone derivatives against methicillin-resistant Staphylococcus aureus. Biol Pharm Bull. 1996, 19(2):311-4.

55. Hu Y, Wang S, Wu X, Zhang J, Chen R, Chen M, Wang Y: Chinese herbal medicine-derived compounds for cancer therapy: A focus on hepatocellular carcinoma. J Ethnopharmacol. 2013, S0378-8741(13)00531-X. 
460

461

462

463

464

465

466

467

468

469

470

471

472

473

474

475

476

477

478

479

480

481

482

483

484

485

486

487

488

56. Han C, Guo J: Antibacterial and anti-inflammatory activity of traditional Chinese herb pairs, Angelica sinensis and Sophora flavescens. Inflammation 2012, 35(3):913-9.

57. Su SY, Hsieh CL: Anti-inflammatory effects of Chinese medicinal herbs on cerebral ischemia. Chin Med. 2011, 6:26.

58. Wang L, Qiu XM, Hao Q, Li DJ: Anti-inflammatory effects of a Chinese herbal medicine in atherosclerosis via estrogen receptor $\beta$ mediating nitric oxide production and NF-kB suppression in endothelial cells. Cell Death Dis. 2013, 4:e551.

59. Jiang L, Deng L, Wu T: Chinese medicinal herbs for influenza. Cochrane Database Syst Rev. 2013, 3:CD004559.

60. Cheng HM, Li CC, Chen CY, Lo HY, Cheng WY, Lee CH, Yang SZ, Wu SL, Hsiang CY, Ho TY: Application of bioactivity database of Chinese herbal medicine on the therapeutic prediction, drug development, and safety evaluation. $J$ Ethnopharmacol 2010, 132(2):429-37.

61. Lu J, Ye S, Qin R, Deng Y, Li CP: Effect of Chinese herbal medicine extracts on cell-mediated immunity in a rat model of tuberculosis induced by multiple drug-resistant bacilli. Mol Med Rep. 2013, 8(1):227-32.

62. Li SZ: Combined traditional Chinese medicine and western medicine in the treatment of 356 cases of scrofula of the neck and axilla. Zhong Xi Yi Jie He Za Zhi 1984, 4(2):90-2.

\section{Tables and Figures}

\section{Figures}

Figure 1: Summary of the data-mining and prioritization approach involving prediction of actives, consensus building and filtering for permeability and undesirable substructures.

Figure 2: Venn diagram showing active molecules filtered by any of the five SMARTS filters.

Figure 3 Top scoring pharmacophore models (magenta color) identified along with the alignment with the input molecules. 


\section{Tables}

490 Table 1. List of 19 compounds predicted as active against non replicating 491 antibiotic tolerant Mycobacterium tuberculosis.

492 Supplementary Data

493 Supplementary Table 1 shows the Chinese molecules used in the present study with 494 their smiles.

495 Supplementary Table 2 shows the molecules predicted to have anti tubercular activity 496 by our models.

497 Supplementary Table 3 shows the 9 molecules which could penetrate the 498 Mycobacterium tuberculosis cell wall.

499 


\section{Figure 1}

Figure1

Summary of the data-mining and prioritization approach involving prediction of actives, consensus building and filtering for permeability and undesirable substructures.

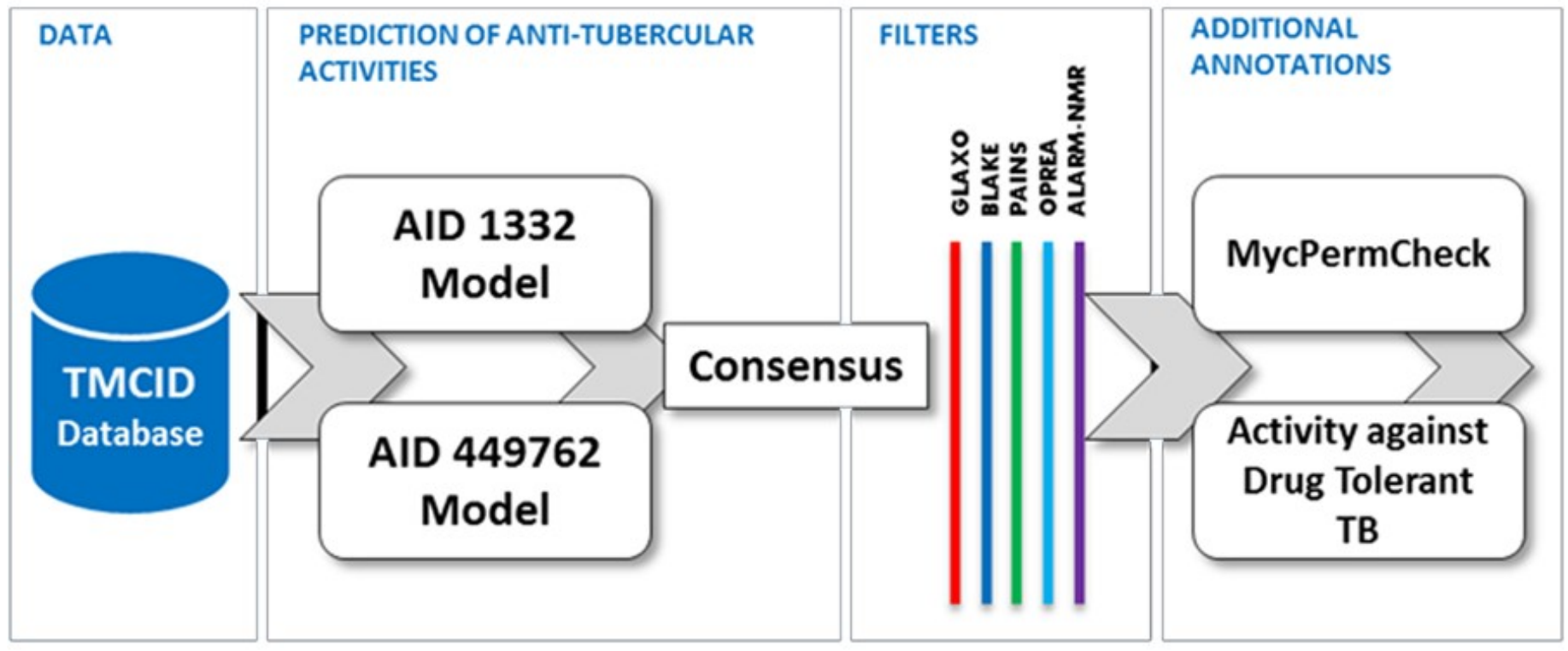




\section{Figure 2}

Figure2

Venn diagram showing active molecules filtered by any of the five SMARTS filters.

Glaxo 1211

Blake 947

PAINS 750

Oprea 738

Alarm NMR 543

Consensus 160

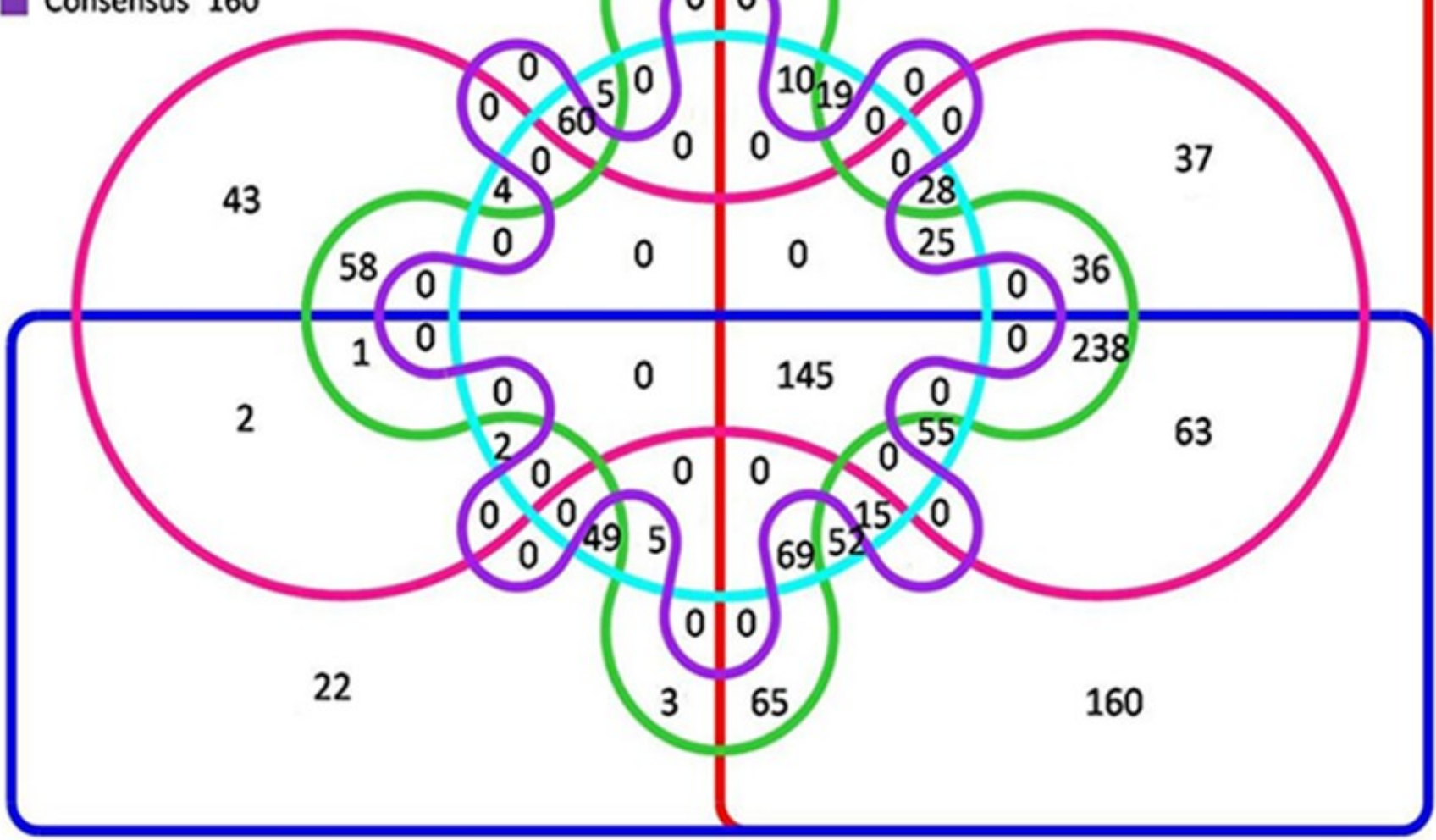




\section{Figure 3}

Figure 3

Top scoring pharmacophore models $(A, B$ and $C)$ identified along with the alignment with the input molecules. The pharmacophores are coloured in magenta.

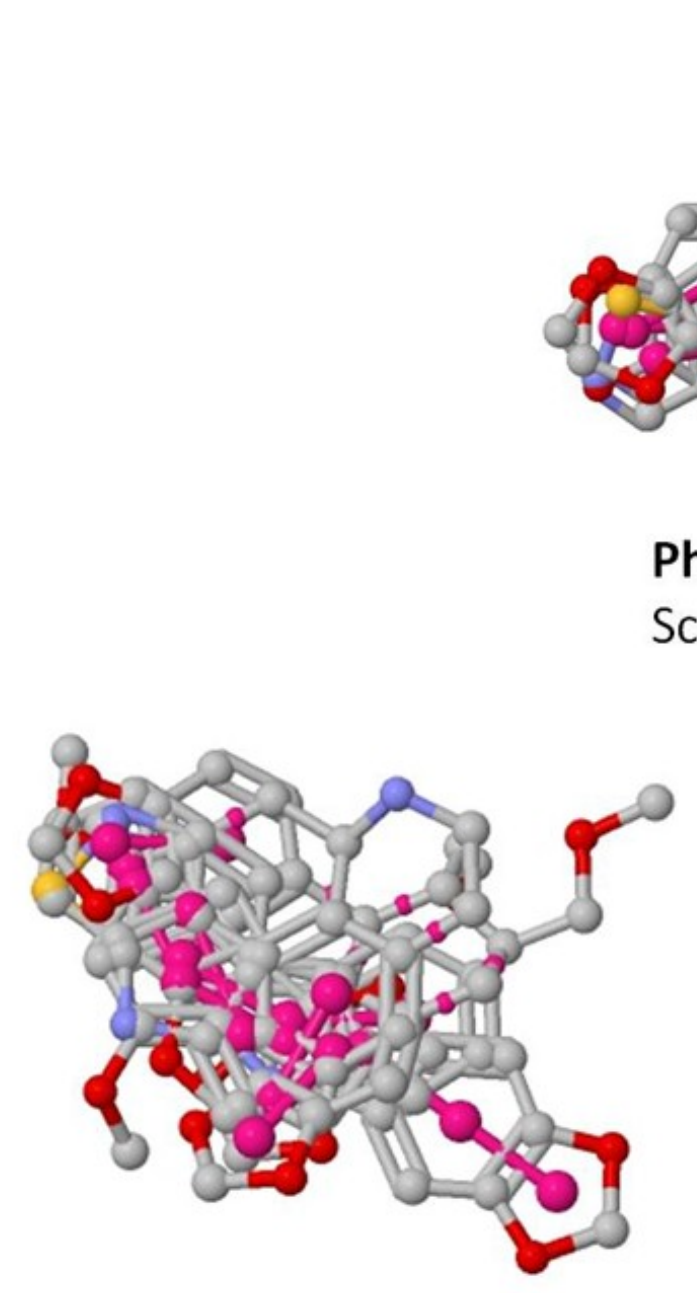

Pharmacophore (B)

Score : 27.78

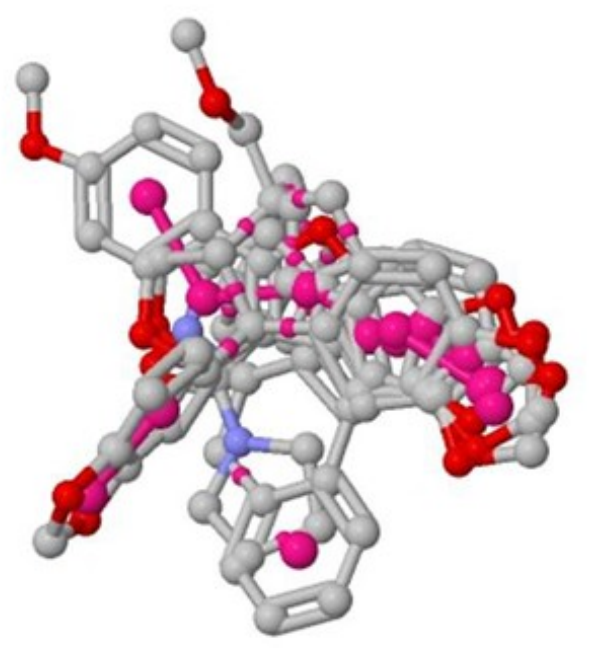

Pharmacophore (C) Score : 25.72 


\section{Table 1 (on next page)}

Table 1

List of 19 compounds predicted as active against non replicating antibiotic tolerant Mycobacterium tuberculosis. 


\begin{tabular}{|c|c|c|c|c|c|c|}
\hline $\begin{array}{l}\text { Comp } \\
\text { ound } \\
\text { No. }\end{array}$ & $\begin{array}{l}\text { Compound } \\
\text { structure }\end{array}$ & Name & \begin{tabular}{|l} 
English \\
Name
\end{tabular} & $\begin{array}{l}\text { Latin } \\
\text { Name }\end{array}$ & \begin{tabular}{|l|} 
Permeabili \\
ty \\
probability
\end{tabular} & \begin{tabular}{|l} 
Sources \\
with \\
antitubercul \\
ar activities
\end{tabular} \\
\hline 1. & & $\begin{array}{l}\text { F } \\
\text { lemichapparin }\end{array}$ & $\begin{array}{l}\text { Climbing } \\
\text { Jewelvine }\end{array}$ & $\begin{array}{l}\text { Derris } \\
\text { scandens }\end{array}$ & 0.993 & \\
\hline 2. & & Murrayafoline a & \begin{tabular}{|l|} 
Taiwan \\
Common \\
Jasminorang \\
e, \\
Indian \\
Common \\
Jasminorang \\
e, \\
Euchretaleaf \\
Common \\
Jasminorang \\
e, \\
Narrowfruit \\
Glycosmis \\
Root \\
\end{tabular} & $\begin{array}{l}\text { Murraya } \\
\text { crenulata, } \\
\text { Murraya } \\
\text { koenigii, } \\
\text { Murraya } \\
\text { euchrestifolia } \\
\text {, Glycosmis } \\
\text { stenocarpa }\end{array}$ & 0.98 & \\
\hline 3. & & $\begin{array}{l}\text { 2-hexenyl } \\
\text { benzoate }\end{array}$ & $\begin{array}{l}\text { Common } \\
\text { Tea, } \\
\text { Szechwan } \\
\text { Tangshen }\end{array}$ & $\begin{array}{l}\text { Camellia } \\
\text { sinensis }, \\
\text { Codonopsis } \\
\text { tangshen }\end{array}$ & 0.855 & \\
\hline 4. & & Anonaine & \begin{tabular}{|l|} 
Hindu Lotus \\
Large \\
Rhizome, \\
Bullockshear \\
t \\
Custardappl \\
e, \\
Custard \\
Apple, \\
\end{tabular} & $\begin{array}{l}\text { Nelumbo } \\
\text { nucifera, } \\
\text { Annona } \\
\text { reticulata, } \\
\text { Annona } \\
\text { squamosa, } \\
\text { Melia } \\
\text { azedarach, } \\
\text { Artabotrys } \\
\end{array}$ & 0.52 & \\
\hline
\end{tabular}




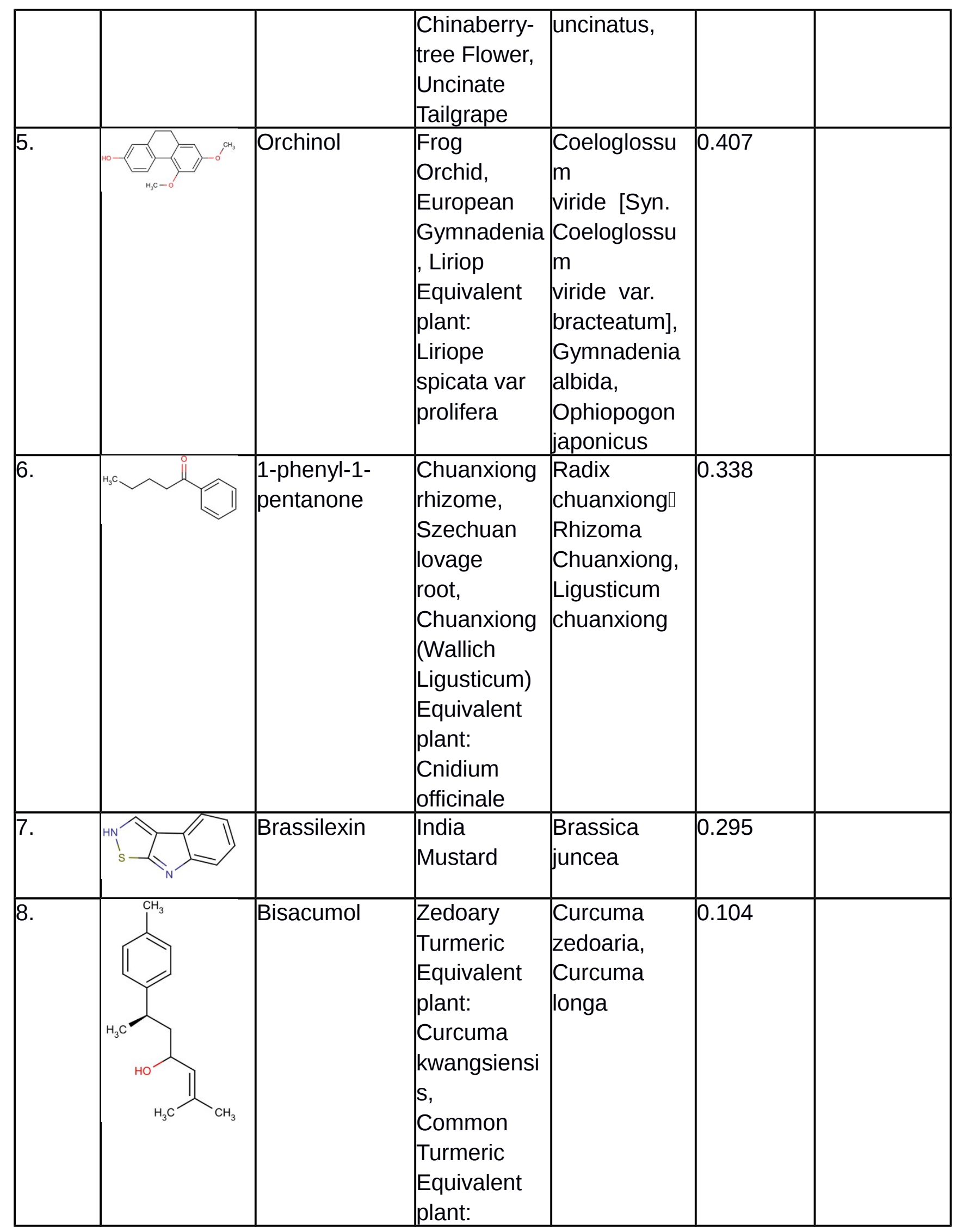




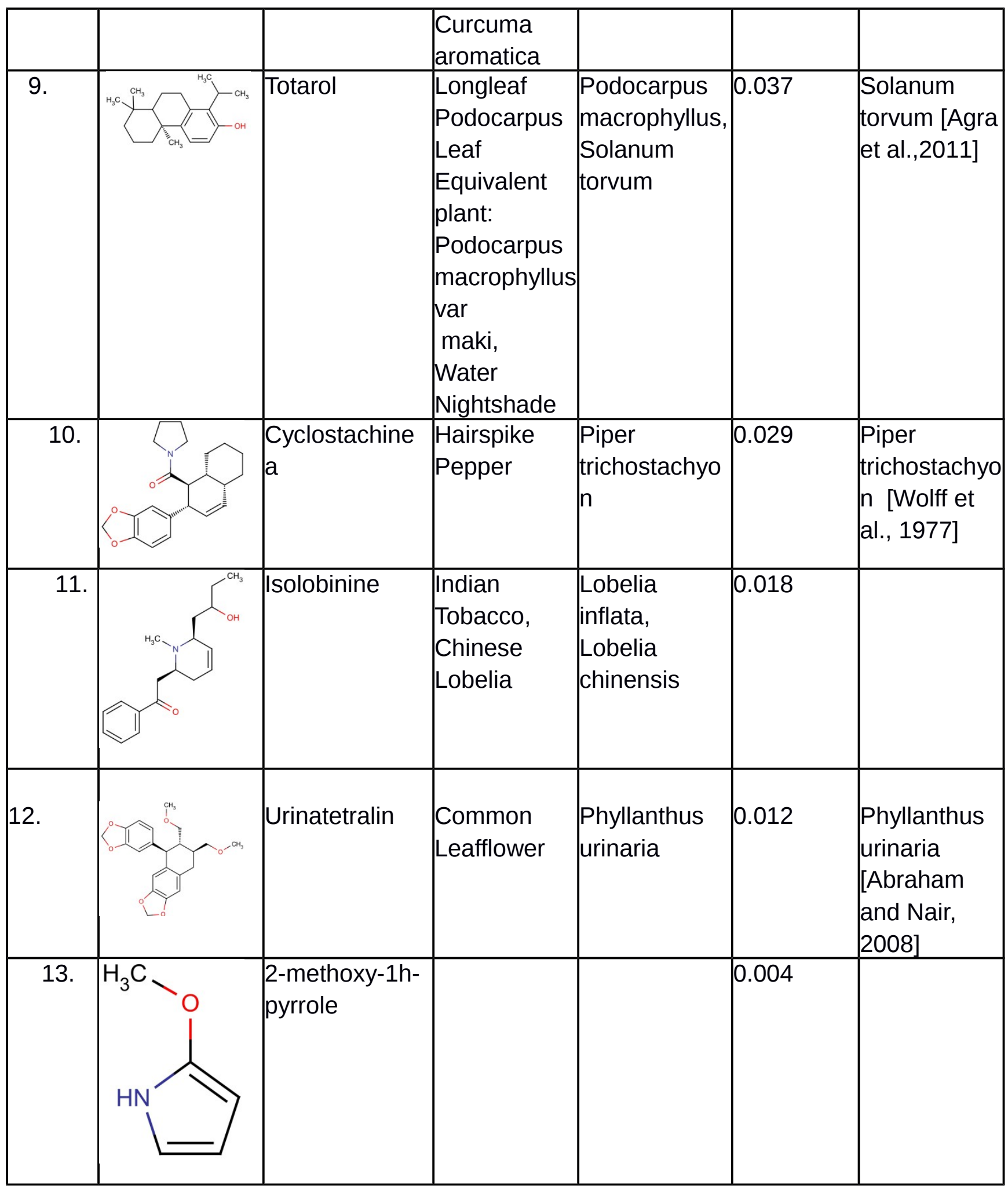




\begin{tabular}{|c|c|c|c|c|c|c|}
\hline 14. & 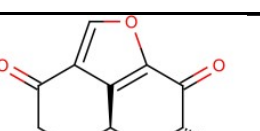 & Gmelofuran & $\begin{array}{l}\text { Medicinal } \\
\text { Breynia Leaf }\end{array}$ & $\begin{array}{l}\text { Breynia } \\
\text { officinalis }\end{array}$ & 0.00 & \\
\hline 15. & & $\begin{array}{l}\text { Petasalbin } \\
\text { methyl ether }\end{array}$ & $\begin{array}{l}\text { Japanese } \\
\text { Butterbur }\end{array}$ & \begin{tabular}{|l|} 
Petasites \\
japonicus
\end{tabular} & 0.00 & \begin{tabular}{|l} 
Petasites \\
japonicus \\
[Fogarty, \\
1990]
\end{tabular} \\
\hline 16. & & Verruculotoxin & & & 0.00 & \\
\hline 17. & & Hinokiol & $\begin{array}{l}\text { Yellowish } \\
\text { Rabdosia }\end{array}$ & $\begin{array}{l}\text { Isodon } \\
\text { flavidus }\end{array}$ & 0.00 & \\
\hline 18. & & Thymine & $\begin{array}{l}\text { Przewalsk } \\
\text { Fritillary, } \\
\text { Anhui } \\
\text { Fritillary, } \\
\text { Ussuri } \\
\text { Fritillary }\end{array}$ & $\begin{array}{l}\text { Fritillaria } \\
\text { przewalskii, } \\
\text { Fritillaria } \\
\text { anhuiensis, } \\
\text { Fritillaria } \\
\text { ussuriensis }\end{array}$ & 0.00 & \begin{tabular}{|l|} 
Fritillaria \\
przewalskii \\
[Chang and \\
Paul, 2001]
\end{tabular} \\
\hline 19. & & \begin{tabular}{|l}
\multicolumn{1}{c}{$\mathrm{n}$ - } \\
methylcorydaldi \\
ne
\end{tabular} & \begin{tabular}{|l|} 
Fendler's \\
Meadowrue, \\
Bracteate \\
Poppy, \\
Asiatic \\
Moonseed \\
Root, \\
Lotusleaftun \\
g \\
\end{tabular} & $\begin{array}{l}\text { Thalictrum } \\
\text { fendleri, } \\
\text { Papaver } \\
\text { bracteatum, } \\
\text { Menispermu } \\
\text { m dauricum, } \\
\text { Hernandia } \\
\text { sonora }\end{array}$ & 0.00 & \begin{tabular}{|l} 
Hernandia \\
sonora \\
[Bourgeois et \\
al., 1999]
\end{tabular} \\
\hline
\end{tabular}

